\title{
Effects of the tool path strategies on incremental sheet metal forming process
}

\author{
L. Ben Said, J. Mars, M. Wali ${ }^{\mathrm{a}}$ And F. Dammak \\ Mechanical Modelisation and Manufacturing Laboratory (LA2MP), National Engineering School of Sfax, \\ B.P W3038, Sfax, University of Sfax, Tunisia
}

Received 19 March 2015, Accepted 13 October 2015

\begin{abstract}
This paper presents a numerical simulation of the incremental sheet metal forming (ISF) process, type single point incremental forming (SPIF). A finite element model (FEM) is developed by using the commercial FE code ABAQUS. An elasto-plastic constitutive model with quadratic yield criterion of Hill'48 and isotropic hardening behavior has been adopted during ISF operation. A user material subroutine (VUMAT) is used to implement this material behavior. Four strategies of tool path during the ISF of a piece having a square box final shape are presented. Results including thickness variation of sheet metal, forming force along $Z$-axis and the Hill' 48 stress distribution for the four strategies are presented and compared at the aim to optimize the SPIF and to see which tool path strategy makes this process more effective with the consideration of the characteristics of specimen manufactured and its material properties.
\end{abstract}

Key words: Incremental sheet forming / numerical simulation / tool path strategy

\section{Introduction}

Incremental sheet forming (ISF) is an innovative process to manufacture sheet metal with numeric increments on CNC machines through plastic deformation. This process is relatively slow, allows the manufacture of prototype parts with complex shapes and high precisions. Two ISF types can be distinguished: the single point incremental forming (SPIF) and the two points incremental forming (TPIF).

The experiences realized with the ISF process are concentrated in the SPIF process using a CNC machine with a forming tool with specific shape. This tool replaces the milling tool and it uses the same mounting elements in $\mathrm{CN}$ machine. Many trajectories are used in incremental forming with numerical increments by the use of CN program in 3 axis or 5 axis machines. The incremental forming tool trajectories are specific to this process and materials of sheets manufactured in ISF.

The literature on the subject of ISF: the integrity of surfaces during and after the incremental forming, the geometry of the specimen manufactured, forming efforts and optimization of forming time are investigated by the majority of researches. The majority of manufactured parts, mentioned in the articles, are not industrial parts but they are test pieces with cone or pyramidal shape where

${ }^{a}$ Corresponding author: mondherwali@yahoo.fr a hemispherical punch is used in the sheet forming process, with a manner close to the operation of a pocket in a CN milling process. So the study of an industrial piece will be significant with good experiment results.

Some studies have been proposed to understand the ISF process by considering different material behavior and various tool paths. Robert et al. [1] presented the implementation of a new algorithm to simulate elastoplastic material behavior with anisotropic plasticity criterion based on the incremental deformation theory in the ABAQUS commercial finite element code. The algorithm is tested for two sheet forming processes: stretch forming, and incremental sheet forming. The aim of the algorithm is to reduce the global CPU time, to analyze the stress state and thickness distribution of the studied sheet metal.

In terms of tool paths, several studies are focused on the development and the optimization of the tool path strategies in ISF process. Concerning tool path generation algorithms, $\mathrm{Lu}$ et al. [2] presented a new featurebased tool path generation algorithm for incremental sheet forming process. In this study, to obtain a better understanding of forming mechanism using the tool path generation method, the thickness distribution, geometric accuracy and surface quality of the ISF formed shapes are compared with the traditional ISF tool path method based on three case studies including a truncated cone with double bottoms, a non-symmetrical cone and a car 
fender. This contribution is based on a conclusion that the conventional contour based strategies have been proven to cause problems in surface quality and geometric accuracy in specimens manufactured by ISF process. Azaouzi and Lebaal [3] showed an optimization procedure tested for a given forming strategy, in order to reduce the manufacturing time and homogenize thickness distribution of an asymmetric part. The forming strategy was determined by finite element analyses in combination with response surface method and sequential quadratic programming algorithm. With their forming strategy they proved that there are contradictory relations between optimal time and homogeneous thickness distribution. Because of conflicting relations between the criteria of sheet metal forming and the solutions that minimize the objective function while preventing that the part thinning does increase and at the same time respecting the part geometry and minimizing the sheet thickness variability. Belchior et al. [4] developed an approach applied in robotized ISF process, this approach consists in coupling a finite element analysis of the ISF (using to predict efforts in this process) with an elastic model of a robot structure where the punch is mounted. The result of finite element simulation was used like input data for the elastic model at the aim to optimize the punch path and to make correction in robotized ISF when the punch makes deviation. The effect of the hardening law (Ludwick or Voce) has been evaluated.

The prototyping of complex sheet metal parts using single point incremental forming (SPIF) requires the generation of optimal tool paths and/or tool path sequences that ensure that the formed part is within geometric design specifications [5]. Behera et al. [5] proposed an analysis methodology using topological conceptual graphs to capture the effects of different phenomena on the final accuracy of a sheet metal part manufactured by SPIF. So they established an algorithm creating partial tool paths when the shape of the sheet formed is too complex and demands sum accuracy. The generation of an optimal tool path depends on the ability to predict the errors using an uncompensated tool path for the original CAD model of the part [6]. Behera et al. [6] presented tool path compensation strategies for single point incremental sheet forming using multivariate adaptive regression splines. The impact of tool path types and other programming parameters on process implementation through an experimental campaign performed on a parallel kinematics machine tool have been discussed in the work of Rauch et al. [7]. The results showed that basic CAM tool paths are not suitable to carry out ISF applications with good efficiency. So they present an approach adapting the tool paths during the manufacturing of a part according to process data evaluations. This new approach is based on a development of intelligent CAM programmed tool paths.

Several researchers have focused their attention on modeling and numerical simulation of the ISF process. Finite element analyses, using an implicit method, have been performed by Ben Ayed et al. [8] to develop a simplified numerical approach to simulate the ISF with precision and with reduction of CPU time. In this contribution, a shell element DKT12 was implemented and coupled with an elasto-plastic model based on a classical flow rule, isotropic hardening has been considered in the simulations.

Different techniques to study the deformation mechanisms in incremental sheet forming and to improve the accuracy of parts have been proposed in literature. Fang et al. [9] presented an investigation on deformation mechanism and fracture behavior in single point incremental forming. Two parameters are considered in their analytic model, bending effect and strain hardening, to describe the localized deformation mechanism. By numerical and experimental investigation they have confirmed that the deformation occurs not only in the contact zone, but also in the neighboring wall which has been already formed in the vicinity of the contact zone. In addition, the fracture tends to appear at the transitional zone between the contact area and the formed wall. They conclude also that the strain hardening has a positive effect on the material formability.

Kurra and Regalla [10] analyzed the formability and thickness distribution in ISF of extra-deep drawing steel. In this work, numerical simulations are performed following the experimental phase to get the thickness distribution using LS-DYNA. The path-independent stress-based forming limit was utilized by Seong et al. [11] taking into account stress-gradient histories through the thickness direction in order to explain more scientific explanation why the incremental sheet forming prevents a neck from initiating and activating. Han et al. [12] studied the springback at the aim to predict and control accurately this factor to design an accurate tool path for ISF. A threedimensional elasto-plastic finite element model was implemented to simulate ISF which is based on the particle swarm optimization neural network.

The present investigation is a contribution of work that started using an incremental sheet metal forming (ISF) process by taking into account the elastoplastic properties with quadratic yield criterion of Hill and isotropic hardening behavior in the model of sheet forming by SPIF. We used the material user subroutine (VUMAT) to implement the material behavior of the aluminum AA1050 based on the recent work of Wali et al. [13] and Mars et al. [14]. Results including thickness distribution, forming forces and the Hill stress distribution can be estimated from this numerical model and compared with the results presented by Ben Ayed et al. [8]. A comparison between four tool paths strategies developed by a known CAM software (CATIA V5 R17) is made to see the effect of these strategies on the ISF parameters and which strategy is more effective for the studied specimen.

\section{Constitutive model}

To model the materials' mechanical behavior, the relationship between the stress and strain should be 
established. It is assumed that a hypoelastic stress-strain relation can be written as

$$
\dot{\sigma}_{i j}=D_{i j k l} \dot{\varepsilon}_{k l}^{e}
$$

where $\dot{\boldsymbol{\sigma}}$ is the material time derivative of the Cauchy stress tensor, $\dot{\varepsilon}^{e}$ is the elastic part of the strain rate tensor and $\boldsymbol{D}$ is the Hooke stress-strain tensor. For isotropic elasticity, tensor $\boldsymbol{D}$ is given in terms of the shear modulus $G$ and Poisson's ratio $\nu$ by

$$
D_{i j k l}=2 G\left(\delta_{i k} \delta_{j l}+\frac{\nu}{1-2 \nu} \delta_{i k} \delta_{j l}\right)
$$

Moreover, the following assumption of strain rate decomposition, is assumed

$$
\dot{\varepsilon}=\dot{\varepsilon}^{e}+\dot{\varepsilon}^{p}
$$

where $\dot{\varepsilon}$ is the strain rate tensor, $\dot{\varepsilon}^{p}$ is the plastic part of the strain rate tensor, and $\dot{\varepsilon}^{e}$ is the elastic part of the strain rate tensor.

Also, we consider the one of the most common yield criteria that is used in the simulation of forming processes, Hill'48 [15] quadratic yield function, which is given, with isotopic hardening, as follow

$$
f=\sqrt{\frac{3}{2}} \varphi(\boldsymbol{\sigma})-\left[\sigma_{y}+R(\kappa)\right], \quad \varphi(\boldsymbol{\sigma})=\sqrt{\boldsymbol{\sigma}^{T} \boldsymbol{P} \boldsymbol{\sigma}}
$$

where $\sigma_{Y}$ is the initial yield stress, $\kappa$ model the isotropic hardening and $\boldsymbol{P}$ is a fourth order tensor which defines the yield criterion. This yield function includes the classical $J_{2}$ plasticity yield condition. The Hill'48 yield criterion, in three-dimensional cases, is obtained by taking

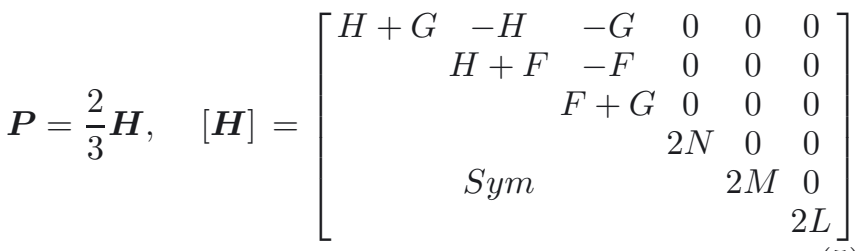

where $F, G, H, N, M$ and $L$ are material constants obtained by tests of the material in different orientations. The $J_{2}$ plasticity yield criterion is recovered using Equation (5) and setting

$$
F=G=H=0.5, \quad N=M=L=1.5
$$

In isotropic elastic material and Hill'48 criterion with plane stress condition, $\boldsymbol{P}$ and $\boldsymbol{D}$ are simply

$$
\begin{aligned}
\boldsymbol{P} & =\frac{2}{3}\left[\begin{array}{ccc}
G+H & -H & 0 \\
-H & F+H & 0 \\
0 & 0 & 2 N
\end{array}\right] \\
\boldsymbol{D} & =\frac{E}{1-\nu^{2}}\left[\begin{array}{ccc}
1 & \nu & 0 \\
\nu & 1 & 0 \\
0 & 0 & (1-\nu) / 2
\end{array}\right]
\end{aligned}
$$

Moreover, with the hypotheses of associated plasticity, the flow rule is given by

$$
\dot{\varepsilon} p=\dot{\gamma} \frac{\partial f}{\partial \boldsymbol{\sigma}}=\sqrt{\frac{3}{2}} \dot{\gamma} \boldsymbol{n}, \quad \boldsymbol{n}=\frac{1}{\varphi} \boldsymbol{P} \boldsymbol{\sigma}
$$

where $\dot{\gamma}$ denotes the plastic multiplier. In Equation (4), $\kappa$ is given by

$$
\dot{\kappa}=-\dot{\gamma} \frac{\partial f}{\partial R}=\dot{\gamma}
$$

Finally, the loading/unloading conditions, formulated in standard Kuhn-Tucker form, are as follows

$$
f \leq 0, \quad \dot{\gamma} \geq 0, \quad \dot{\gamma} f=0
$$

\subsection{Integration algorithm}

The plastic strain $\varepsilon_{n+1}^{p}$ is determined by integration of the flow rule over a time step. The integration is made by using the implicit backward Euler's method which makes the algorithm unconditionally stable. This leads to

$$
\varepsilon_{n+1}^{p}=\varepsilon_{n}^{p}+\sqrt{3 / 2} \Delta \gamma \boldsymbol{n}_{n+1}
$$

where

$$
\boldsymbol{n}_{n+1}=\frac{1}{\varphi_{n+1}} \boldsymbol{P} \boldsymbol{\sigma}_{n+1}
$$

Using Equations (3) and (11) into the stress-strain relation, Equation (1), gives the stress tensor as

$$
\boldsymbol{\sigma}_{n+1}=\boldsymbol{\sigma}^{t r}-\sqrt{3 / 2} \Delta \gamma \boldsymbol{D} \cdot \boldsymbol{n}_{n+1}
$$

where $\boldsymbol{\sigma}^{t r}$ is the elastic trial stress

$$
\boldsymbol{\sigma}^{t r}=\boldsymbol{D} \cdot\left(\varepsilon_{n+1}-, \varepsilon_{n}^{p}\right) \quad \varepsilon_{n+1}=\varepsilon_{n}+\nabla^{s} \Delta \boldsymbol{u}
$$

with Equations (12) and (13), the stress tensor, $\boldsymbol{\sigma}_{n+1}$, can then be computed as

$$
\boldsymbol{\sigma}_{n+1}=\boldsymbol{I}_{c}^{-1} \cdot \boldsymbol{\sigma}^{t r}, \quad \boldsymbol{I}_{c}=\boldsymbol{I}+\sqrt{\frac{3}{2}} u \boldsymbol{D} \boldsymbol{P}, \quad u=\frac{\Delta \gamma}{\varphi_{n+1}}
$$

Inserting this relation into the yield condition, Equation (4), renders the following algorithmic consistency condition as

$$
f_{n+1}=\sqrt{\frac{3}{2}} \varphi_{n+1}-\sigma_{p, n+1}=0
$$

with

$$
\varphi_{n+1}=\left[\boldsymbol{\sigma}^{t r T} \cdot \boldsymbol{I}_{c}^{-T} \cdot \boldsymbol{P} \cdot \boldsymbol{I}_{c}^{-1} \boldsymbol{\sigma}^{t r}\right]^{1 / 2}, \sigma_{p}=\sigma_{Y}+R
$$

This furnishes a non linear scalar equation which will be solved with the Newton method. If the Newton iteration is used to solve the yield equation, the derivative of the yield function is needed. At the $k$ iteration, this is given by

$$
\frac{\mathrm{d} f_{k}}{\mathrm{~d}(\Delta \gamma)}=\sqrt{3 / 2} \frac{\mathrm{d} \varphi_{k}}{\mathrm{~d}(\Delta \gamma)}-R^{\prime}
$$


Table 1. Integration algorithm .

(I) Compute trial elastic stress

$\boldsymbol{\sigma}^{t r}=\boldsymbol{D} \cdot\left(\varepsilon_{n+1} \varepsilon_{n}^{p}\right)$

(II) Find $\Delta \gamma$ by local iteration

(i) $f_{k}=\sqrt{\frac{3}{2}} \varphi_{k}-\sigma_{p, k}, \quad \varphi_{k}=\left[\boldsymbol{\sigma}^{t r T} \cdot \boldsymbol{I}_{c}^{-T} \cdot \boldsymbol{P} \cdot \boldsymbol{I}_{c}^{-1} \cdot \boldsymbol{\sigma}^{t r}\right]^{1 / 2}$

$\mathbf{I}_{c}=\mathbf{I}+u \sqrt{\frac{3}{2}} \mathbf{D} \mathbf{P}, \quad u=\frac{\Delta \gamma}{\varphi_{k}}$

(ii) $f_{k}^{\prime}=\sqrt{3 / 2} \frac{\mathrm{d} \varphi_{k}}{\mathrm{~d}(\Delta \gamma)}-\sigma_{p, k}^{\prime}$

(iii) $\Delta \gamma_{k+1}=\Delta \gamma_{k}-\frac{f_{k}}{f_{k}^{\prime}}$

(iv) if $\left|f_{k}\right|>$ Tol then $k=k+1$ go to (i)

(III) Update variables

$\boldsymbol{\sigma}_{n+1}=\mathbf{I}_{c}^{-1} \cdot \boldsymbol{\sigma}^{t r}$

Remark : It is possible to incorporate the discussed model into an eight-node solid-shell finite element formulation by using $\boldsymbol{P}$ Equation (5) [16] and can be successfully applied in metal forming simulations. Furthermore, there exist also fournode shell element formulations which are applicable to thin shell structures by using $\boldsymbol{P}$ Equation (7) [17].

with, see Wali et al. [13],

$$
\begin{gathered}
\frac{\mathrm{d} \varphi}{\mathrm{d}(\Delta \gamma)}=\boldsymbol{n} \cdot \boldsymbol{I}_{c}^{-1}\left[-\sqrt{\left.\frac{3}{2} \varphi u^{\prime} \boldsymbol{D} \boldsymbol{n}\right]}\right. \\
u^{\prime}=\sqrt{3 / 2}\left[\frac{\sigma_{p}-R^{\prime} \Delta \gamma}{\sigma_{p}^{2}}\right]
\end{gathered}
$$

The solution of Equation (16) may then be effectively accomplished by the simple local iteration procedure and the whole integration algorithm is given in Table 1.

\subsection{Extension to large deformation elastoplasticity}

In the case of large deformation elastoplasticity, with a hypoelastic-based approach, Equation (1) will be replaced by a linear relationship of an arbitrary objective rate of the Kirchhoff stress $\tau$ and the elastic rate of deformation tensor $\boldsymbol{d}^{e}$. When considering the Green-Naghdi rate as in ABAQUS/Explicit, with no essential elastic volume change $(\boldsymbol{\tau} \approx \boldsymbol{\sigma})$, this leads to

$$
\boldsymbol{\sigma}^{o}=\dot{\boldsymbol{\sigma}}-\boldsymbol{\Omega} \boldsymbol{\sigma}+\boldsymbol{\sigma} \boldsymbol{\Omega}=\boldsymbol{D} \boldsymbol{d}^{e}
$$

where $\Omega$ is a skew-symmetric second-order Eulerian quantity given by

$$
\boldsymbol{\Omega}=\dot{\boldsymbol{R}} \boldsymbol{R}^{T}
$$

and $\boldsymbol{R}$ is the rotation tensor of the polar decomposition of the deformation gradient $\boldsymbol{F}=\boldsymbol{v} \boldsymbol{R}=\boldsymbol{R} \boldsymbol{U}$. The integration algorithm defines the integration of the stress tensor associated with the material behavior as

$$
\boldsymbol{\sigma}_{n+1}=\boldsymbol{\sigma}^{t r} \sqrt{3 / 2} \Delta \gamma \boldsymbol{D} \boldsymbol{n}_{n+1}
$$

with

$$
\boldsymbol{\sigma}^{t r}=\Delta \boldsymbol{R} \boldsymbol{\sigma}_{n} \Delta \boldsymbol{R}^{T}+\boldsymbol{D} \Delta \boldsymbol{d}
$$

Table 2. Mechanical properties of the aluminum AA1050 material.

\begin{tabular}{cccccc}
\hline \multicolumn{2}{c}{ Elastic Prop. } & \multicolumn{4}{c}{ Hill'48 Coefficients } \\
\hline$E(\mathrm{GPa})$ & $\nu$ & $F$ & $G$ & $H$ & $N$ \\
\hline 70 & 0.33 & 0.706 & 0.662 & 0.338 & 1.253 \\
\hline
\end{tabular}

where $\Delta \boldsymbol{R}$ is the increment of rotation tensor and $\Delta \boldsymbol{d}$ is the strain increment. $\Delta \boldsymbol{R}$ and $\Delta \boldsymbol{d}$ are calculated in a suitable way. Equations (22) and (23) can then be used as Equation (13) and the rest of development remains the same. The presented model is implemented as user material subroutine (VUMAT) into the commercial finite element code ABAQUS/Explicit.

\section{Results and discussions}

In this section, square box application of ISF was investigated and the results carried out with the use of two numerical treatments, the first is based on ABAQUS/Explicit code and the second is based on the user material subroutine (VUMAT). The material used in all numerical tests is the aluminum alloy AA1050 material. The elastic properties and Hill' 48 coefficients of this material are from Ben Ayed et al. [8] and given in Table 2.

The isotropic hardening behavior is modeled by the Swift law

$$
\sigma_{p}(\boldsymbol{\kappa})=119.5(0.000142+\boldsymbol{\kappa})^{0.235}
$$

The initial dimension of the sheet is $200 \times 200 \times 1.5 \mathrm{~mm}$. The punch tool has a diameter of $10 \mathrm{~mm}$, assumed as a rigid body and no property is assigned. The frustum of the square box has a depth of $10 \mathrm{~mm}$ and a base area of $80 \times 80 \mathrm{~mm}$. In the results given with Hill criterion, it is assumed that the axes of anisotropy are the axes of reference, thus making $x$ the rolling direction and $y$ the transverse direction of the sheet metal. The friction coefficient between punch tool and sheet metal is assumed to be 0.1 [8]. The sheet is meshed using 2728 triangular elements S3R in ABAQUS/Explicit with five integration points through the thickness.

The tool paths and the dimensions of the desired shape are shown in Figure 1 which presents the first strategy named strategy (A) developed by Ben Ayed et al. [8]. This strategy (A) is first examined and constitutes a validation test. Then, three other strategies, named (B), $(\mathrm{C})$ and $(\mathrm{D})$, are presented and compared with the strategy $(\mathrm{A})$.

\subsection{Validation test, strategy (A)}

The first tool path strategy during the ISF of the square box is presented in Figure 1. In this strategy the path is formed by an ensemble of square contour $(80 \times 80)$ vertically incremented with $1 \mathrm{~mm}$ to achieve a depth of $10 \mathrm{~mm}$. 


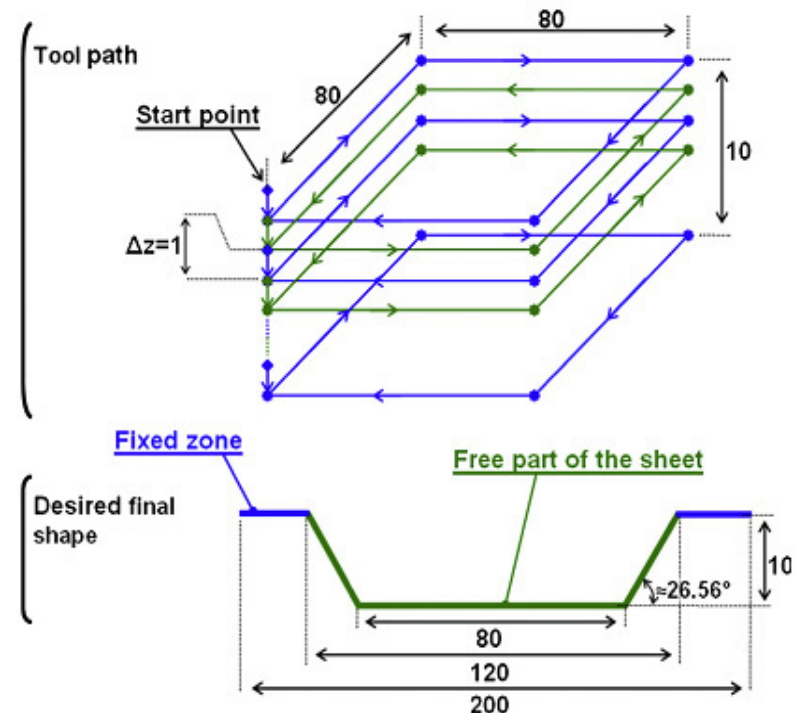

Fig. 1. Tool path and the final shape of incremental formed sheet [8].

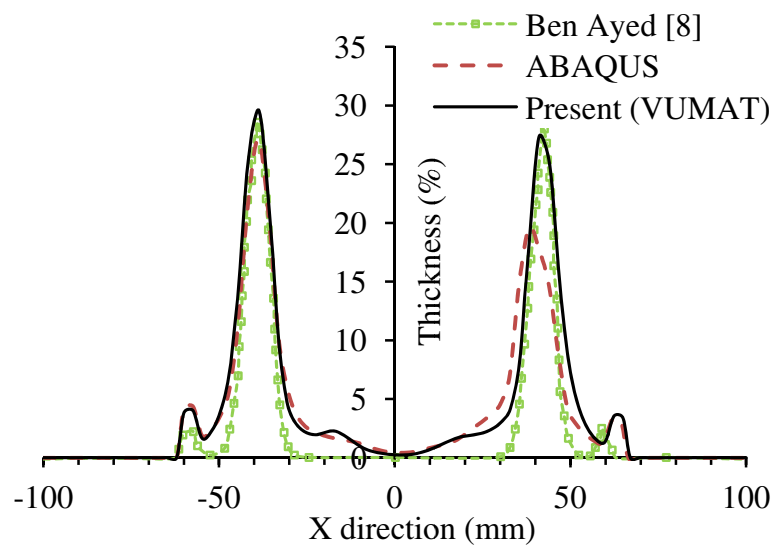

Fig. 2. Thickness variation in the cut plane $Y=0$.

The thickness variation along the cut plane, $Y=0$ is presented in Figure 2, presenting a comparison between the results carried out from simulations using ABAQUS/Explicit code, user material subroutine (VUMAT) and the results presented by Ben Ayed et al. [8].

According to the thickness evolution, significant deformations of square box are located principally in the vicinity of punch path for the three results. The maximum variation of thickness evolution carried out by the use of user material subroutine (VUMAT) is similar to results presented by Ben Ayed et al. [8]. However, a difference is looked in the center of sheet. Thickness variation in this zone is caused by the tensile stress created during the movement of tool in the square box contour. Results carried out by the use of ABAQUS/Explicit present a nonsymmetrical evolution compared to the two other results.

The evolution of the vertical forming force during the ISF process of the studied sheet is illustrated in Figure 3. The vertical forming force due to the contact pressure

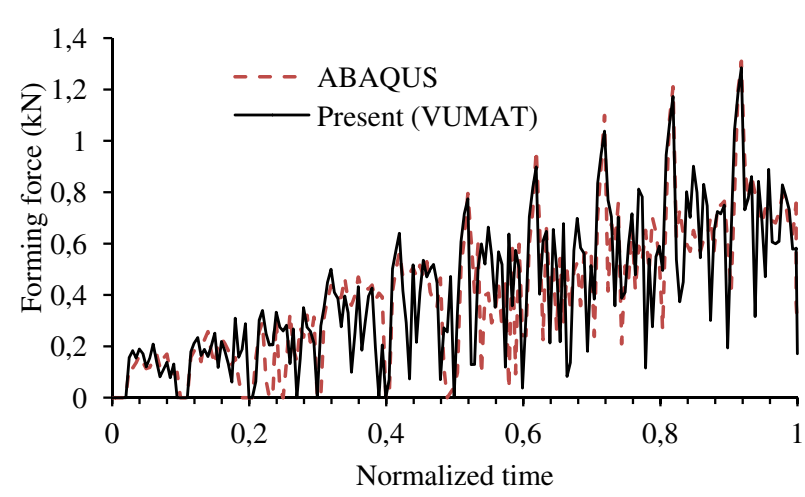

Fig. 3. Evolution of the forming force $F_{z}(\mathrm{~N})$.

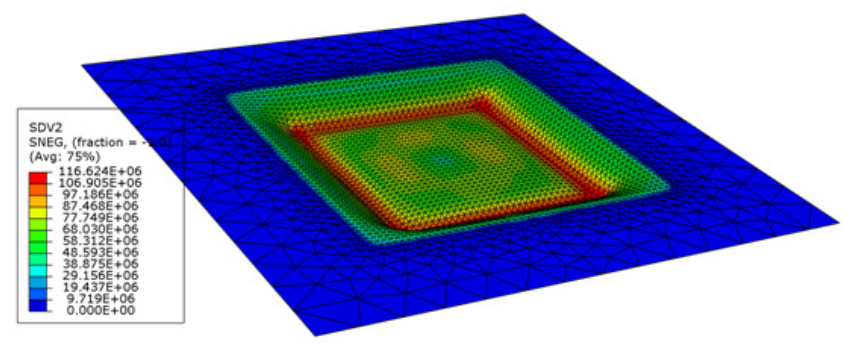

Fig. 4. Distribution of Hill'48 stress using VUMAT.

reaches the maximum value in the last pass of the punch when incremental forming is in a depth of $10 \mathrm{~mm}$. The value of this effort is in the same range of efforts recognized in the majority of articles established by many research groups, e.g. $[2,4]$. Result carried out from the user material subroutine (VUMAT) is in good agreement with the one obtained by ABAQUS/Explicit, even the same range of maximum effort is recognized.

The distribution of the Hill' 48 stress in the deformed zone under the action of the punch during the ISF process is shown in Figure 4 which is the result of simulation using the user material subroutine (VUMAT). According to this figure the major Hill'48 stress is localized under the punch tool, located principally along the punch path.

\subsection{Effects of the tool path strategies}

A lot of researches have been established in the area of strategies definition in CNC milling process which can be compared to the ISF process by using CNC machines. In the conventional tool path generation algorithms, tool paths are generated according to the sliced contours in the horizontal plane. This kind of contour traces lines of equal altitude in the $Z$ direction. Using the CAM software CATIA V5R17, three other strategies are developed defining the punch path during the incremental forming of the same application described previously. Figure 5 presents the three tool path strategies, (B), (C) and (D). In spite of the considerable progress of technology implemented, the CAM software offers only derivative strategies from standard or conventional one experienced as the zigzag 


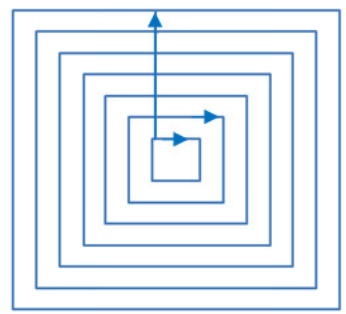

(a)

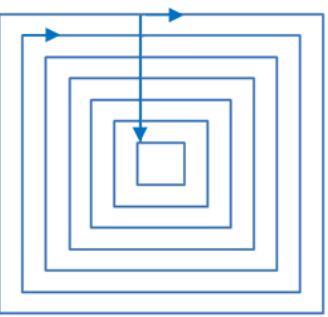

(b)

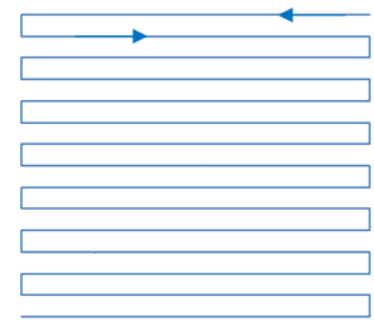

(c)

Fig. 5. Three tool path strategies developed by CATIA V5R17.

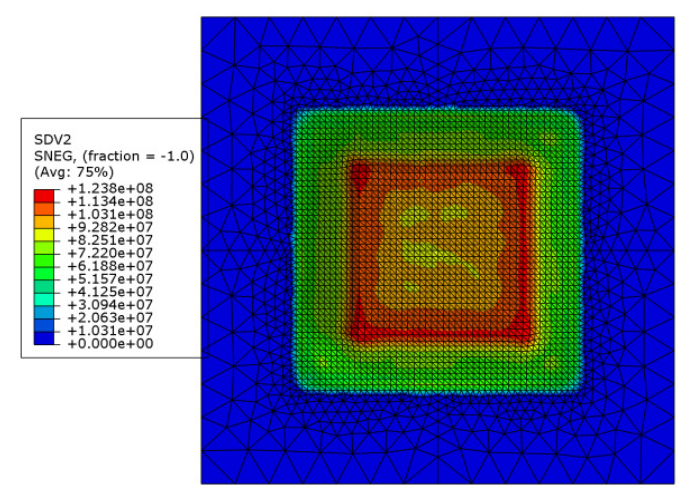

(a)

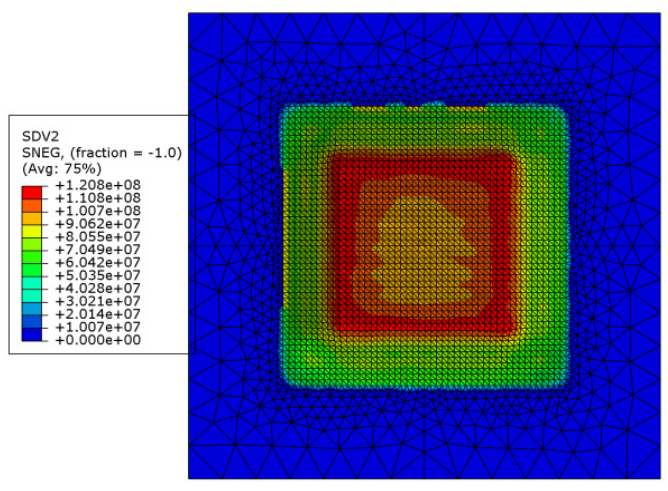

(b)

Fig. 6. Hill'48 stress distribution (a) strategy (A), (b) strategy (B).

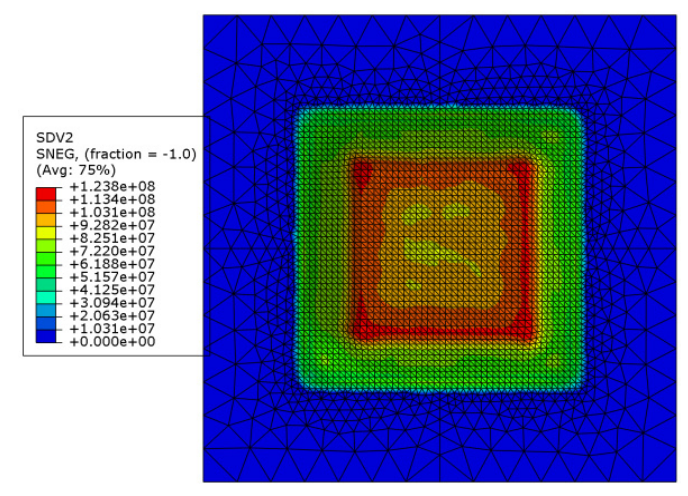

(a)

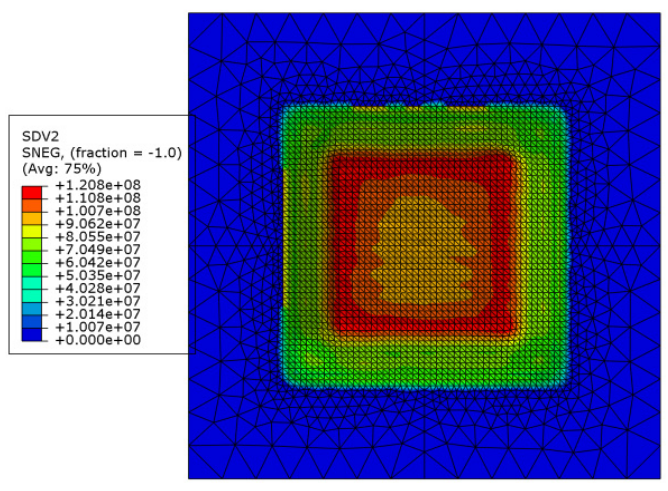

(b)

Fig. 7. Hill'48 stress distribution (a) strategy (C), (b) strategy (D).

(D) and parallel contour (B) and (C). CAM software can optimize these strategies according to the shape and the precision of studied parts.

The Hill'48 stress distributions obtained using the four strategies, (A), (B), (C) and (D), after the ISF operation, are shown in Figures 6 and 7 . According to these figures, changes in the tool path strategies have a direct impact on the Hill'48 stress distributions. By the use of these CAM strategies the localized maximum stress can be eliminated. Also, the D-strategy seems the best choice in terms of Hill'48 stress distribution.

To identify the influence of tool path strategy used in ISF process, numerical results, obtained with the use of user material subroutine (VUMAT) for four tool path strategies, have been compared in terms of maximum of vertical force $F_{z-\max }$ and thickness evolution. Figure 8 illustrates the evolution of the maximum vertical force due to the contact pressure for the four tool path strategies, and the thickness variation along the cut plane $Y=0$ is presented in Figure 9. Also, the thickness distributions in the sheet surface for the four studied strategies are presented in Figures 10 and 11.

Results carried out from these figures show clearly that the three strategies (B), (C) and (D) are more effective in the ISF manufacturing compared to the strategy (A). The strategies (B) and (C) present the lower 


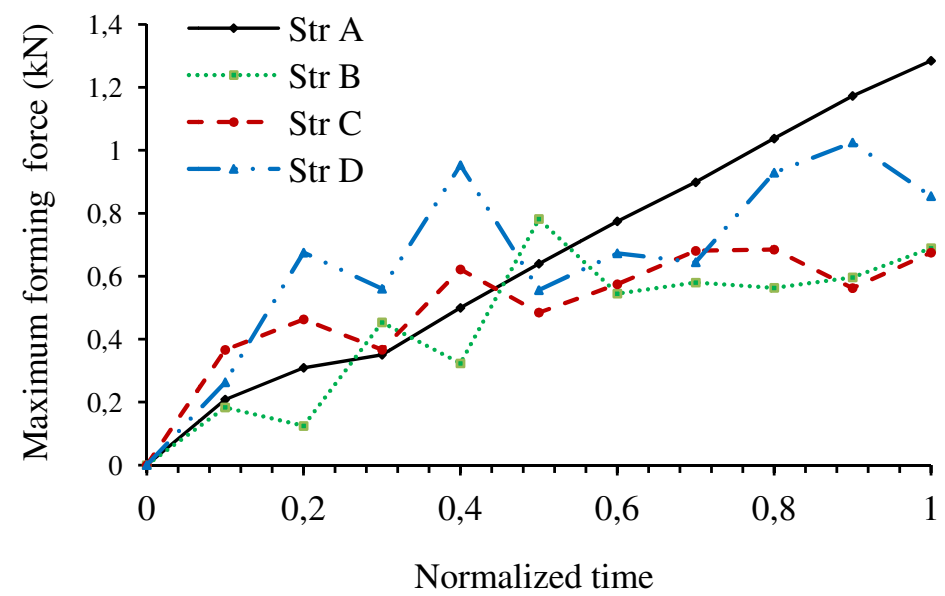

Fig. 8. Evolution of the maximum forming force $F_{z-\max }$ for the four strategies.

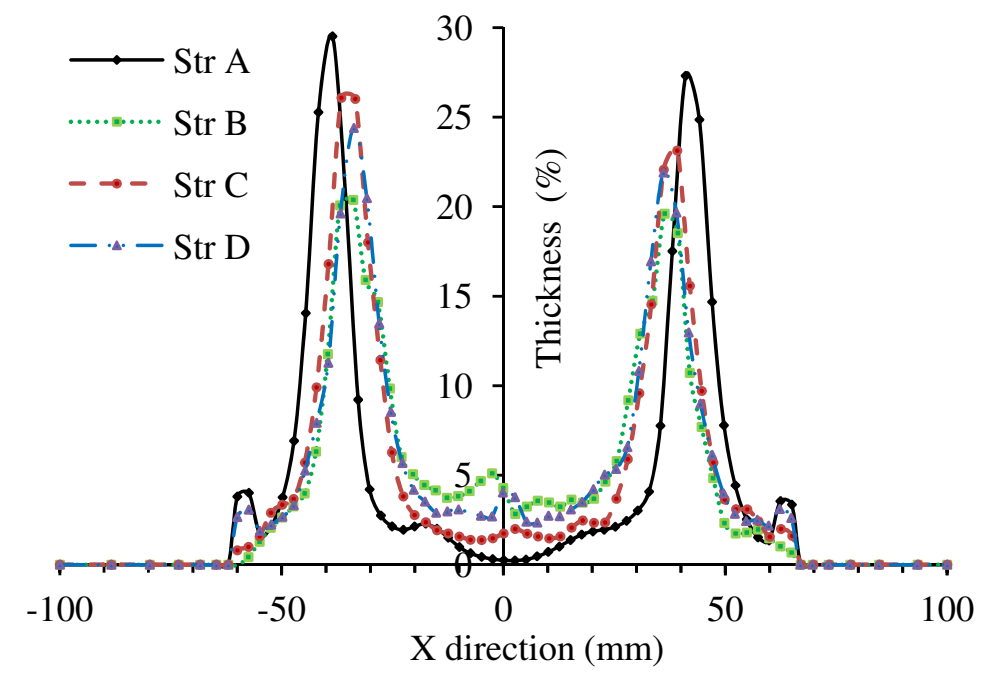

Fig. 9. Thickness variation in the cut plane $Y=0$ for the four strategies.

values of vertical force during the ISF operation. This vertical force has a direct impact on the roughness of the manufactured part.

From Figures 9-11, it is noted that the thickness distributions are not symmetric and significant deformations are located principally along the contour of the square box base for the four strategies. But we can note that the use of strategy (B) decreases greatly the thickness variation of the manufactured part.

Table 3 illustrates the CPU time for the tool path strategies (A), (B), (C) and (D). The CPU time was reduced when we used the strategy (D) called Zig-Zag tool path. This type of strategy proved good results in other manufacturing process like pocket milling in term of productivity to the extent that the tool path is the shortest compared to other strategies. An experimental study will allow us to verify these results for sheet metal incremental forming application.
Table 3. CPU Time for strategies (A), (B), (C) and (D).

\begin{tabular}{cccc}
\hline$(\mathrm{A})$ & $(\mathrm{B})$ & $(\mathrm{C})$ & $(\mathrm{D})$ \\
\hline $03: 32: 02$ & $03: 39: 01$ & $03: 55: 25$ & $02: 35: 37$ \\
\hline
\end{tabular}

\section{Conclusion}

In this paper, finite element model is developed, based on Hill'48 yield criterion and isotropic hardening behavior, simulating an example of single point incremental sheet forming. The material model is implemented by the use of user material subroutines (VUMAT) to predict ISF process. A comparison between four tool paths strategies on the SPIF of a square box application shows that there are significant differences between the results carried out for the four strategies. These results show that the use of known CAM software developing sum standard tool paths strategies can be used in ISF applications (best CPU time, 


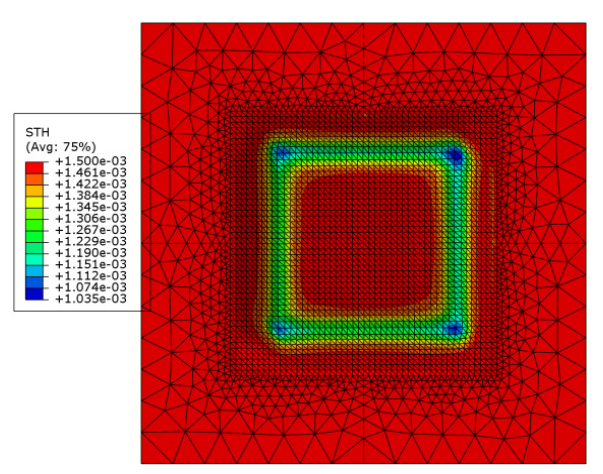

(a)

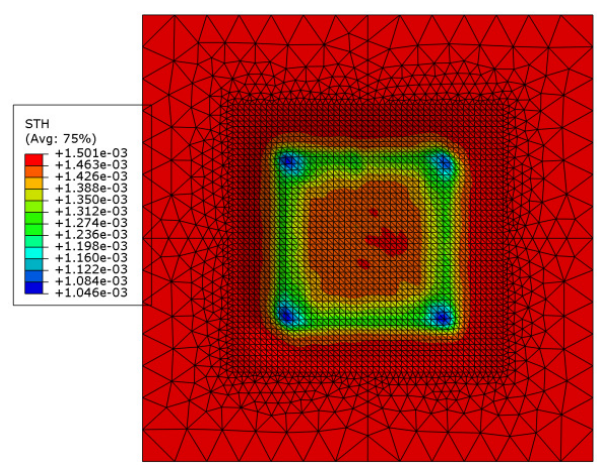

(b)

Fig. 10. Thickness distribution (a) strategy (A), (b) strategy (B).

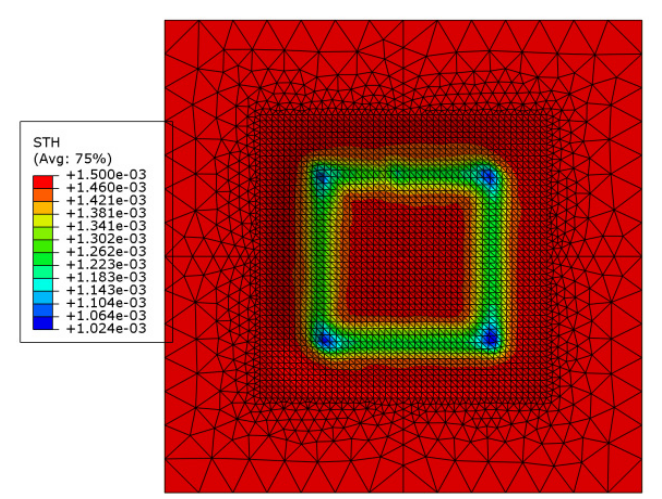

(a)

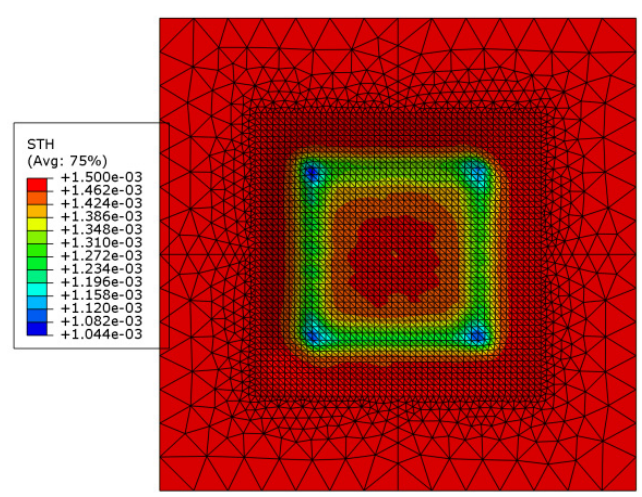

(b)

Fig. 11. Thickness distribution (a) strategy (C), (b) strategy (D).

good thickness homogeneity...), compared with results carried out by the use of a strategy $(\mathrm{A})$

Also we intend to make experimental investigation in this theme and compare results with numerical simulations, optional parameters will be added to the current ISF application in order to study other tests of the incremental sheet forming process.

\section{References}

[1] C. Robert, A. Delamézière, P. Dal Santo, J.L. Batoz, Comparison between incremental deformation theory and flow rule to simulate sheet-metal forming processes, J. Mater. Process. Techol. 212 (2012) 1123-1131

[2] B. Lu, J. Chen, H. Ou, J. Cao, Feature-based tool path generation approach for incremental sheet forming process, J. Mater. Process. Tech. 213 (2013) 1221-1233

[3] M. Azaouzi, N. Lebaal, Tool path optimization for single point incremental sheet forming using response surface method, Simul. Modell. Practice Theory 24 (2012) 49-58

[4] J. Belchior, L. Leotoing, D. Guines, E. Courteille, P. Maurine, A Process/Machine coupling approach: Application to Robotized Incremental Sheet Forming, J. Mater. Process. Techol. 214 (2014) 1605-1616
[5] A.K. Behera, B. Lauwers, J.R. Duflou, Tool path generation framework for accurate manufacture of complex 3D sheet metal parts using single point incremental forming, Comput. Ind. 65 (2014) 563-584

[6] A.K. Behera, J. Verbert, B. Lauwers, J. R. Duflou, Tool path compensation strategies for single point incremental sheet forming using multivariate adaptive regression splines, Comput.-Aid. Design 45 (2013) 575-590

[7] M. Rauch, J.Y. Hascoet, J.C. Hamann, Y. Plenel, Tool path programming optimization for incremental sheet forming applications, Comput.-Aid. Design 41 (2009) $877-885$

[8] L. Ben Ayed, C. Robert, A. Delamézière, M. Nouari, J.L. Batoz, Simplified numerical approach for incremental sheet metal forming process, Eng. Struct. 62-63 (2014) $75-86$

[9] Y. Fang, B. Lu, J. Chen, D.K. Xu, H. Ou, Analytical and experimental investigations on deformation mechanism and fracture behavior in single point incremental forming, J. Mater. Process. Techol. 214 (2014) 1503-1515

[10] S. Kurra, S.P. Regalla, Experimental and numerical studies on formability of extra-deep drawing steel in incremental sheet metal forming, J. Mater. Res. Technol. 3 (2014) 158-171

[11] D.Y. Seong, M.Z. Haque, J.B. Kim, T.B. Stoughton, J.W. Yoon, Suppression of necking in incremental sheet forming, Int. J. Solids Struct. 51 (2014) 2840-2849 
[12] F. Han, J. MO, H. Qi et al., Springback prediction for incremental sheet forming based on FEM-PSONN technology, Trans. Nonferrous Met. Soc. China 23 (2013) 10611071

[13] M. Wali, H. Chouchene, L. Ben Said, F. Dammak, One-equation integration algorithm of a generalized quadratic yield function with Chaboche non-linear isotropic/kinematic hardening, Int. J. Mech. Sci. 92 (2015) 223-232

[14] J. Mars, M. Wali, A. Jarraya, F. Dammak, A. Dhiab, Finite element implementation of an orthotropic plasticity model for sheet metal in low velocity impact simulations, Thin Wall. Struct. 89 (2015) 93-100
[15] R. Hill, A theory of the yielding and plastic flow of anisotropic metals, Proc. Roy. Soc. London A 193 (1948) $281-297$

[16] A. Hajlaoui, A. Jarraya, I. Kallel-Kamoun, F. Dammak, Buckling analysis of a laminated composite plate with delaminations using the enhanced assumed strain solid shell element, J. Mech. Sci. Technol. 26 (2012) 3213-3221

[17] F. Dammak, S. Abid, A. Gakwaya, G. Dhatt, A formulation of the nonlinear discrete Kirchhoff quadrilateral shell element with finite rotations and enhanced strains, Revue Européenne des Elements Finis 14 (2005) 7-31 